\title{
Analysis of treatment effect of urinary kallidinogenase combined with edaravone on massive cerebral infarction
}

\author{
$\mathrm{JIANG} \mathrm{KE}^{1}$ and $\mathrm{MOU} \mathrm{JING}^{2}$ \\ ${ }^{1}$ Department of Neurology, East People's Hospital, Xuzhou, Jiangsu 221000; ${ }^{2}$ Department of \\ Cardiology, Xuzhou City Center Hospital, Xuzhou, Jiangsu 221009, P.R. China \\ Received February 10, 2016; Accepted May 23, 2016
}

DOI: $10.3892 /$ br.2016.692

\begin{abstract}
The aim of the study was to investigate the clinical effect of urinary kallidinogenase combined with edaravone in the treatment of massive cerebral infarction. A total of 58 patients with massive cerebral infarction were admitted to hospital between January 2013 and January 2014. There were 34 male and 24 female patients. The patients were randomly divided into the observation and control groups ( $n=29$ cases per group). The patients in the control group received edaravone treatment, while patients in the observation group were treated with urinary kallidinogenase and edaravone. The clinical effects of the two groups were then compared. The results showed that the National Institutes of Health Stroke Scale score and serum C-reactive protein level of the patients in the two groups were significantly decreased following treatment. The decreased degree in the observation group was significantly smaller than that in the control group. The difference was statistically significant $[(11.03 \pm 3.75)$ vs. $(16.58 \pm 7.43)$ scores, $\mathrm{P}<0.05$; $(9.88 \pm 4.82)$ vs. $(11.98 \pm 4.69) \mathrm{mmol} / \mathrm{l}, \mathrm{P}<0.05]$. The serum levels of vascular endothelial growth factor were significantly increased in patients of the two groups after treatment. The increased degree in the observation group was significantly higher than that in the control group. The difference was statistically significant [(268.51 \pm 77.34$)$ vs. $(188.82 \pm 57.33) \mathrm{ng} / 1, \mathrm{P}<0.05]$. The total effective rate of the observation group was significantly higher than that of the control group and the difference was statistically significant (89.66 vs. $62.07 \%, \mathrm{P}<0.05)$. In conclusion, urinary kallidinogenase combined with edaravone treatment has a certain clinical curative effect on massive cerebral infarction.
\end{abstract}

\section{Introduction}

Cerebral infarction is a common disease of the central nervous system, accounting for $75-80 \%$ of cerebrovascular disease,

Correspondence to: Dr Mou Jing, Department of Cardiology, Xuzhou City Center Hospital, 199 South Jiefang Road, Xuzhou, Jiangsu 221009, P.R. China

E-mail: siyuanmujing@126.com

Key words: massive cerebral infarction, urinary kallidinogenase, edaravone, clinical effect with a high rate of morbidity and mortality (1). Family history of cerebral infarction, age, diabetes and atherosclerosis were some of the major risk factors of cerebral infarction. The morbidity of cerebral infarction in men $>55$ years or women $>60$ years of age was much higher than that of other age groups (2). Massive cerebral infarction occurs primarily due to the cerebral arterial main trunk block that causes greater tissue damage range and a higher degree of risk (3).

Urinary kallidinogenase and edaravone are a new selective cerebral vasodilator and oxygen-free radical scavenger, which have recently been used for the treatment of cerebral infarction and are considered effective (4).

In the present study, the application of urinary kallidinogenase and edaravone for the treatment of massive cerebral infarction was assessed to enhance the clinical curative effect of massive cerebral infarction.

\section{Materials and methods}

Cases. A total of 58 patients with massive cerebral infarction were admitted to the Hospital between January 2013 and January 2014, and were examined and diagnosed using computed tomography (CT) or magnetic resonance imaging (MRI). Patients with systemic complications, severe bleeding tendency, severe liver and kidney dysfunction, other serious organic diseases, drug allergy in the study, and cerebral hemorrhage identified by CT or MRI examination were excluded. Patients or their families participated voluntarily, were provided with information regarding the study, and provided written informed consent. The study was approved by the ethics committee of Xuzhou City Center Hospital. The patients were randomly divided into the observation and control groups, with 29 cases in each group. In total, 18 men and 11 women were included in the observation group. The patient age range was 44-80 years, with an average age of $53.67 \pm 7.24$ years. Complications identified included 22 cases of hypertension and 12 cases of diabetes. The control group subjects included 16 men and 13 women, with an age range of 45-78 years, and an average age of 54.13 \pm 8.22 years. Complications for the control group included 21 cases of hypertension and 10 cases of diabetes. No significant differences were identified between the two groups with regard to age, gender, and complications $(\mathrm{P}>0.05)$, making these parameters comparable. 
Table I. Changes of serum VEGF and hs-CRP in patients of the two groups before and after the treatment.

\begin{tabular}{lcccccc}
\hline \multirow{2}{*}{ Groups } & & \multicolumn{2}{c}{$\operatorname{VEGF}(\mathrm{ng} / \mathrm{l})$} & & \multicolumn{2}{c}{ hs-CRP $(\mathrm{mmol} / \mathrm{l})$} \\
\cline { 3 - 4 } & Cases & Before treatment & After treatment & & Before treatment & After treatment \\
\hline Observation & 29 & $155.72 \pm 53.54$ & $268.51 \pm 77.34^{\mathrm{a}, \mathrm{b}}$ & & $15.02 \pm 4.61$ & $9.88 \pm 4.82^{\mathrm{a}, \mathrm{b}}$ \\
Control & 29 & $154.45 \pm 52.19$ & $188.82 \pm 57.33^{\mathrm{a}}$ & & $14.97 \pm 4.56$ & $11.98 \pm 4.69^{\mathrm{a}}$ \\
\hline
\end{tabular}

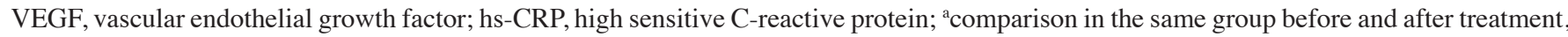
$\mathrm{P}<0.05$; ${ }^{\mathrm{b}}$ comparison of the observation group and the treatment group, $\mathrm{P}<0.05$.

Table II. Comparison of the NIHSS scores of two groups before and after treatment (mean \pm standard deviation).

\begin{tabular}{lccc}
\hline Groups & Cases & Before treatment & After treatment \\
\hline Observation & 29 & $22.93 \pm 8.29$ & $11.03 \pm 3.75^{\mathrm{a}, \mathrm{b}}$ \\
Control & 29 & $22.67 \pm 7.24$ & $16.58 \pm 7.43^{\mathrm{a}}$
\end{tabular}

NIHSS, National Institutes of Health Stroke Scale; ${ }^{\text {accmparison of }}$ the group before and after treatment, $\mathrm{P}<0.05$; ${ }^{\mathrm{b}}$ comparison between the observation and treatment groups, $\mathrm{P}<0.05$.

Method. The patients in the two groups received basic treatment, in the form of oral administration of aspirin (300 mg/day), which was reduced to $100 \mathrm{mg} /$ day after 10 days of continuous medication and oral atorvastatin, $20 \mathrm{mg}$ once daily. Blood glucose and blood pressure were controlled for patients with hypertension and hyperglycemia. However, use of angiotensin converting enzyme inhibitors of antihypertensive drugs were disallowed during the application of urinary kallidinogenase. Patients in the control group received edaravone $30 \mathrm{mg}+100 \mathrm{ml} 0.9 \% \mathrm{NaCl}$ injection solution by intravenous drip, four times daily. Subsequently, 0.15 PNA dissolved in $0.9 \% 100 \mathrm{ml} \mathrm{NaCl}$ solution was intravenously administered once daily in the observation group on the basis of treatment of the control group. Patients in the two groups were treated for 14 days as a course of treatment.

Observation index. Venous blood samples of the two groups were collected prior to 14 days after treatment, respectively. The vascular endothelial growth factor (VEGF) level was detected with the application of ELISA, and the serum high-sensitivity C-reactive protein (hs-CRP) level was analyzed with immune turbidimetry following conventional centrifugal separation. Adverse reactions of the two groups were observed during the treatment.

Liver and renal function and blood and urine routine examination were conducted prior to treatment and 14 days after treatment, respectively.

Standard of curative effect. 'Scoring criteria of clinical neurological deficit for patients with stroke' set by the Chinese Society for Neuroscience were used to assess patients with neurological deficits and the curative effect according to the National Institutes of Health Stroke Scale (NIHSS) (5). Scoring was carried out as indicated by NIHSS: Basic cure, reduction by $91-100 \%$ with a clinical disability degree of level 0 ; marked improvements, decreased by $46-90 \%$ with a clinical disability degree of level 1-3; progress, reduction by 18-45\%; no change, decrease or increase of $<17 \%$; and deterioration, increase of $>18 \%$. The total efficiency was shown as cases of (basic cure + marked improvement + progress $) /$ total cases $\mathrm{x} 100 \%$.

Statistical analysis. Data were analyzed using SPSS 18.0 statistics software (IBM SPSS, Armonk, NY, USA). Measurement data were presented as mean \pm standard deviation using the t-test. Enumeration data were presented by rate (\%) using the $\chi^{2}$ test. $\mathrm{P}<0.05$ was considered to indicate a statistically significant difference.

\section{Results}

Changes of serum VEGF and hs-CRP before and after the treatment. Serum VEGF and hs-CRP levels of the two groups had no significant difference before treatment $(\mathrm{P}<0.05)$. Serum VEGF levels in the two groups were significantly increased after treatment and serum VEGF in the observation group was significantly higher than that of the control group. hs-CRP of the two groups was significantly decreased and hs-CRP of the observation group was significantly lower than those of the control group $(\mathrm{P}<0.05$, Table I).

Changes of NIHSS score before and after treatment. NIHSS scores of the two groups were not significantly different before treatment $(\mathrm{P}>0.05)$. NIHSS scores of the two groups were significantly decreased after treatment $(\mathrm{P}<0.05)$. NIHSS scores of the observation group were significantly lower than that of the control group $(\mathrm{P}<0.05$, Table II).

\section{Clinical curative effect}

Adverse reactions. One patient in the observation group had a mild decrease of blood pressure and was relieved by symptomatic treatment. The control group showed no adverse reaction. Blood and urine routine, liver, and kidney function had no obvious abnormalities in the two groups before and after treatment. No significant differences of adverse drug reaction were observed between the two groups ( $\mathrm{P}>0.05$, Table III).

\section{Discussion}

Massive cerebral infarction refers mainly to complete stroke of the middle cerebral artery, carotid artery, or cerebral cortical 
Table III. Total effective rate of the observation group was significantly higher than that of the control group.

\begin{tabular}{|c|c|c|c|c|c|c|c|c|}
\hline Groups & Cases & $\begin{array}{l}\text { Basic cure } \\
\quad(\mathrm{n}, \%)\end{array}$ & $\begin{array}{c}\text { Significant } \\
\text { progress } \\
(\mathrm{n}, \%)\end{array}$ & $\begin{array}{l}\text { Progress } \\
(\mathrm{n}, \%)\end{array}$ & $\begin{array}{c}\text { No change } \\
\quad(\mathrm{n}, \%)\end{array}$ & $\begin{array}{l}\text { Deterioration } \\
\quad(\mathrm{n}, \%)\end{array}$ & $\begin{array}{l}\text { Death } \\
(\mathrm{n}, \%)\end{array}$ & $\begin{array}{c}\text { Total effective } \\
\text { rate } \\
(\mathrm{n}, \%)\end{array}$ \\
\hline Observation & 29 & 8 (27.59) & $11(37.93)$ & $8(27.59)$ & $2(6.90)$ & $0(0.00)$ & $0(0.00)$ & $26(89.66)^{\mathrm{a}}$ \\
\hline Control & 29 & $4(13.79)$ & $8(27.59)$ & $6(20.69)$ & $9(31.03)$ & $2(6.90)$ & $0(0.00)$ & $18(62.07)$ \\
\hline
\end{tabular}

${ }^{a}$ Comparison between the observation group and the treatment group, $\mathrm{P}<0.05$.

branches and is mainly marked by progressive contralateral gaze palsy, contralateral complete limb hemiplegia, and unilateral sensory disturbance (6). It usually shows obvious brain edema and intracranial hypertension at the same time and may develop into brain herniation $(7,8)$. Drug treatment is one of the means used for the clinical treatment of massive cerebral infarction. Thrombolytic therapy is considered an effective method for the treatment of ultra-early cerebral infarction (9). However, it has a limited therapeutic time window. Thrombolytic therapy can achieve noteworthy results only when used within 3.0-4.5 h of occurence (9). Cerebral infarction also tends to be acute. Thus, the use of thrombolytic therapy is always accompanied with high risk and its application is restricted $(8,10-12)$. Previous findings have shown that only $<3 \%$ of the massive cerebral infarction patients can receive thrombolytic therapy in a timely manner. However, most patients did not undergo thrombolytic treatment $(13,14)$. Therefore, it is imperative to identify a more effective therapy with longer treatment time.

In the early stage of cerebral infarction, the brain tissue in the cerebral infarction region experiences metabolic acidosis due to severe hypoxia ischemia. Intracellular calcium overloads significantly, generating a large amount of oxygen-free radical. Oxygen-free radicals are thought to be the main physiological and pathological basis of cerebral infarction $(15,16)$. Ischemia reperfusion injury occurred after cerebral infarction. Lipid soluble radical, hydroxyl radical, and superoxide anion-based toxic-free radicals are produced, which directly cause oxidative damage of intracellular lipid, protein, and nucleic acid and can mediate an ischemic cascade reaction to induce neuronal cell apoptosis and edema $(17,18)$. Therefore, the key to the treatment of large area cerebral infarction lies in improving ischemia, inhibiting, and scavenging oxygen-free radicals.

Edaravone is a new type of antioxidant and free radical scavenger. It has potent hydroxyl radical, active oxygen molecule scavenging ability, and anti-lipid peroxidation effect. Consequently, it can inhibit the cerebral edema and brain tissue injury process it involved $(11,14)$. In addition, it can effectively alleviate the degree of injury of vascular endothelial cells of the ischemic region, improving the neuronal cell hypoxia tolerance to alleviate the local cerebral edema and reduce the infarct. Edaravone has an inhibitory effect on delayed neuronal apoptosis, which can effectively protect the patients' nerve function $(19,20)$. Blood-brain barrier permeability for edaravone is $\sim 60 \%$. Its intravenous injection can effectively remove cytotoxic hydroxyl-free radicals and effectively inhibit the irreversible damage to nucleic acid, and protein damage mediated by peroxyl radical (16-18). Kimura et al (18) administered the auxiliary treatment of edaravone to 40 cases of patients with massive cerebral infarction and compared that treatment type with conventional symptomatic treatment. The results showed that the NIHSS scores were significantly lower at 14 and 28 days after the auxiliary treatment. The total effective rate was $75 \%$, which was significantly higher than that of the conventional symptomatic treatment (47.5\%), confirming its positive effect. Urinary kallidinogenase is tissue human urinary kallikrein extracted from self-urine, which can catalyze the hydrolysis of kininogen and generate kallidin, selectively dilating ischemic artery blood flow, improving the ischemic brain tissue and effectively improving local microcirculation, thereby reducing the infarct $(11,14)$. In addition, it can improve the effective rates of the organization utilization for glucose to improve tissue glucose metabolism, and inhibit platelet aggregation and blood coagulation to promote effectively the infarct vascular regeneration $(11,19,20)$. Two-drug combination has a synergistic effect based on the mechanism of the two drugs, which is expected to improve the treatment effect.

In the present study, the patients in the observation group were treated with urinary kallidinogenase and edaravone combined with traditional treatment, while patients in the control group were administered edaravone and routine treatment. The NIHSS score in the observation group was significantly lower than that of the control group after 14 days of treatment. At the same time, the total effective rate of the observation group was $89.66 \%$, which was significantly higher than $62.07 \%$ of the control group. One case in the observation group showed a mild decrease of blood pressure during treatment with no other adverse reactions and good safety.

In summary, urinary kallidinogenase combined with edaravone can improve the nerve function defect of patients in the treatment of massive cerebral infarction with less adverse reaction and obvious curative effect. Thus, it is a safe, reliable and attractive treatment type and worthy of application.

\section{References}

1. Busing KA, Schulte-Sasse C, Fluchter S, Suselbeck T, Haase KK, Neff W, Hirsch JG, Borggrefe $M$ and Duber C: Cerebral infarction: Incidence and risk factors after diagnostic and interventional cardiac catheterization-prospective evaluation at diffusion-weighted MR imaging. Radiology 235: 177-183, 2005.

2. Lindley RI, Wardlaw JM, Sandercock PA, Rimdusid P, Lewis SC, Signorini DF and Ricci S: Frequency and risk factors for spontaneous hemorrhagic transformation of cerebral infarction. J Stroke Cerebrovasc Dis 13: 235-246, 2004. 
3. Hosomi N, Sueda Y, Masugata H, Dobashi H, Murao K, Ueno M, Miki T, Kohno M, Nishiyama A and Matsumoto M: The optimal timing of antihypertensive medication administration for morning hypertension in patients with cerebral infarction. Hypertens Res 35: 720-724, 2012.

4. Fukuta T, Asai T, Sato A, Namba M, Yanagida Y, Kikuchi T, Koide H, Shimizu K and Oku N: Neuroprotection against cerebral ischemia/reperfusion injury by intravenous administration of liposomal fasudil. Int J Pharm 506: 129-137, 2016.

5. Zhang C, Tao W, Liu M and Wang D: Efficacy and safety of human urinary kallidinogenase injection for acute ischemic stroke: A systematic review. J Evid Based Med 5: 31-39, 2012.

6. Subramaniam S and Hill MD: Massive cerebral infarction. Neurologist 11:150-160, 2005.

7. Camerlingo M, Valente L, Tognozzi M, Beretta GL, Moschini L and Cesana BM: C-reactive protein levels in the first three hours after acute cerebral infarction. Int J Neurosci 121: 65-68, 2011.

8. Lee BJ, Egi Y, van Leyen K, Lo EH and Arai K: Edaravone, a free radical scavenger, protects components of the neurovascular unit against oxidative stress in vitro. Brain Res 1307: 22-27, 2010.

9. Zhang PL, Wang YX, Chen Y, Zhang CH, Li CH, Dong Z, Yin $\mathrm{H}$, Zhang FF and Wang JH: Use of intravenous thrombolytic therapy in acute ischemic stroke patients: Evaluation of clinical outcomes. Cell Biochem Biophys 72: 11-17, 2015.

10. Jiao L, Zhang J, Li Z, Liu H, Chen Y and Xu S: Edaravone alleviates delayed neuronal death and long-dated cognitive dysfunction of hippocampus after transient focal ischemia in Wistar rat brains. Neuroscience 182: 177-183, 2011.

11. Isahaya K, Yamada K, Yamatoku M, Sakurai K, Takaishi S, Kato B, Hirayama T and Hasegawa Y: Effects of edaravone, a free radical scavenger, on serum levels of inflammatory biomarkers in acute brain infarction. J Stroke Cerebrovasc Dis 21: 102-107, 2012.

12. Huang $\mathrm{G}$ and $\mathrm{Xu} \mathrm{W}$ : Edaravone may be an efficacious adjunctive therapeutic agent for decompression sickness: An editorial perspective. Undersea Hyperb Med 40: 215-217, 2013.
13. Ahmad A, Khan MM, Javed H, Raza SS, Ishrat T, Khan MB, Safhi MM and Islam F: Edaravone ameliorates oxidative stress associated cholinergic dysfunction and limits apoptotic response following focal cerebral ischemia in rat. Mol Cell Biochem 367: $215-225,2012$.

14. Nakase T, Yoshioka S and Suzuki A: Free radical scavenger, edaravone, reduces the lesion size of lacunar infarction in human brain ischemic stroke. BMC Neurol 11: 39, 2011.

15. Kikuchi K, Tancharoen S, Takeshige N, Yoshitomi M, Morioka M, Murai Y and Tanaka E: The efficacy of edaravone (radicut), a free radical scavenger, for cardiovascular disease. Int J Mol Sci 14: 13909-13930, 2013.

16. Liu XY, Yao LL, Chen YJ, Tao BB, Yu YC, Bian WH, Yu J and Wang YG: Survivin is involved in the anti-apoptotic effect of edaravone in PC12 cells. Mol Cell Biochem 327: 21-28, 2009.

17. Yan Y, Gong K, Ma T, Zhang L, Zhao N, Zhang X, Tang P and Gong Y: Protective effect of edaravone against Alzheimer's disease-relevant insults in neuroblastoma N2a cells. Neurosci Lett 531: 160-165, 2012.

18. Kimura K, Aoki J, Sakamoto Y, Kobayashi K, Sakai K, Inoue T, Iguchi Y and Shibazaki K: Administration of edaravone, a free radical scavenger, during t-PA infusion can enhance early recanalization in acute stroke patients - a preliminary study. J Neurol Sci 313: 132-136, 2012.

19. Jian Z, Yong R, Chengliang Z, Yu S and Chen WH: Preparation and physicochemical characteristics of the complex of edaravone with hydroxypropyl- $\beta$-cyclodextrin. Carbohydr Polym 83: 1101-1105, 2011

20. Oster S, Radad K, Scheller D, Hesse M,Balanzew W, Reichmann H and Gille G: Rotigotine protects against glutamate toxicity in primary dopaminergic cell culture. Eur J Pharmacol 724: 31-42, 2014. 